\title{
Wenn Schweigen gefährlich ist
}

\author{
David Schwappach ${ }^{a}$, Katrin Gehring ${ }^{b}$
}

${ }^{a}$ Prof. Dr., MPH, Wissenschaftlicher Leiter Stiftung Patientensicherheit Schweiz; ${ }^{b}$ Dr., Wissenschaftliche Mitarbeiterin Stiftung Patientensicherheit Schweiz

\section{Zusammenfassung}

Wenn in der Patientenversorgung etwas nicht gut läuft oder es zu Fehlern kommt, bemerken dies die beteiligten Pflegefachpersonen, Ärztinnen und Ärzte häufig zuerst. Um die Patientensicherheit zu gewährleisten, ist es zentral, dass sie diese Bedenken direkt äussern. Wir untersuchten, ob und wie Mitarbeitende in der Onkologie ihre Kollegen auf Fehler oder riskante Verhaltensweisen ansprechen und welche Faktoren dies erklären. Die Ergebnisse zeigen, dass Mitarbeitende beider Berufsgruppen häufig Situationen erleben, die Nachfragen, Hinweise und Abklärung erfordern. Ärzte und Pflegende benutzen oft Gesten und Mimik, um Kollegen Sicherheitshinweise zu geben. In der Onkologie gibt es bereits eine gut etablierte Kultur zur Kommunikation in Bezug auf die Medikationssicherheit. In anderen Bereichen fällt das Ansprechen von Sicherheitsbedenken aber deutlich schwerer, z.B. bei Hygienemassnahmen oder im Kontext invasiver Prozeduren. Gerade jüngere und tieferrangige Mitarbeitende wägen genau ab, ob und wie sie Kollegen oder Vorgesetzte auf ein Risiko hinweisen, und halten Bedenken eher zurück. Der Motivation, die Sicherheit der Patienten zu gewährleisten, steht die Sorge gegenüber, soziale Beziehungen zu gefährden, Kollegen blosszustellen oder Patienten zu verunsichern. Die Kultur in der jeweiligen Abteilung ist ein wichtiger Faktor, der das Schweigen über Sicherheitsbedenken erklärt.

\section{Einleitung}

Wie in allen Bereichen der Medizin stellen unerwünschte Ereignisse auch in der Onkologie eine Gefahr für Patienten dar. Die Onkologie ist ein multiprofessionell geprägter und hochkomplexer Bereich der Gesundheitsversorgung, in dem bereits einzelne, kleinste Fehler zu schwerwiegenden Schädigungen führen können. Die Kommunikation in onkologischen Teams und die Frage, ob und wie Mitarbeitende konkrete Sicherheitsbedenken äussern und auf die Einhaltung von Sicherheitsregeln drängen ("speak up»), ist ein wichtiges Element für die Patientensicherheit und für die Entwicklung einer Sicherheitskultur. Solches "speaking up" kann beschrieben werden als verbindliche Kommunikation von Sicherheitsbedenken durch
Informationen, Fragen, Einschätzungen oder Meinungsäusserungen in klinischen Situationen, in denen es akuten Handlungsbedarf gibt, damit möglicher Schaden vom Patienten abgewendet werden kann [1]. Ziel für die Gesundheitsversorgung muss es sein, dass Sicherheitsbedenken ausgesprochen und gehört werden. Fehlermeldesysteme und Root-Cause-Analysen zeigen allerdings, dass Spitalmitarbeitende zwar häufig Fehler, riskante Verhaltensweisen oder übergangene Sicherheitsregeln im Team bemerken, aber diese Beobachtungen oft für sich behalten und nicht direkt ansprechen. Damit geht ein grosses Potential für den Schutz der Patienten vor Schädigungen verloren. Umso wichtiger ist es, zu verstehen, welche Faktoren und Bedingungen dazu beitragen, dass Ärzte und Pflege-

\section{Jüngere und tieferrangige Mitarbeitende} wägen genau ab, ob und wie sie Kollegen oder Vorgesetzte auf ein Risiko hinweisen, und halten Bedenken eher zurück.

fachpersonen ihre Bedenken gegenüber Kollegen klar kommunizieren. In unserer Studie untersuchten wir Erfahrungen, Barrieren und unterstützende Faktoren, die ein solches Eintreten der Mitarbeitenden für die Patientensicherheit in konkreten Situationen beeinflussen. Wir gingen der Frage nach, wie häufig Ärztinnen, Ärzte und Pflegefachpersonen in der Onkologie Sicherheitsbedenken haben, ob und wie sie ihre Kollegen auf Fehler oder riskante Verhaltensweisen ansprechen und welche Faktoren dies erklären.

\section{Methode}

An der Studie beteiligten sich neun onkologische Abteilungen von Deutschschweizer Spitälern. Darunter waren Universitäts-, Kantons- und Regionalspitäler, stationäre und ambulante Abteilungen sowie pädiatrische onkologische Kliniken. Die Studie beinhaltete qualitative Interviews mit Ärztinnen, Ärzten und Pflegefachpersonen, die in der onkologischen Patientenversorgung tätig sind, sowie eine schriftliche Befragung. In den persönlichen, semi-strukturierten Interviews wurden Fachpersonen verschiedener Hierarchiestufen zu ihren persönlichen Erfahrungen und Erlebnissen befragt. Es wurde erhoben, in welchen Situationen sie Sicherheitsbedenken haben, wie sie zu 
einer Entscheidung kommen, wie sie sich verhalten, ob und wie sie ihre Bedenken äussern und welche Reaktionen sie von Kollegen und Vorgesetzten bislang erfahren haben. Insgesamt wurden 32 Interviews mit Pflegefachleuten und Ärzten von onkologischen Stationen und Ambulatorien geführt, die verschriftlicht und dann durch zwei Experten unabhängig voneinander mit einer inhaltsanalytischen Methode kodiert (offene Kodierung) und ausgewertet wurden. Der schriftliche Fragebogen richtete sich an alle Mitarbeitenden der teilnehmenden Abteilungen. Dafür wurde ein Fragebogen entwickelt, der aus zwei Teilen bestand: Im allgemeinen Teil wurden die Häufigkeit von Sicherheitsbedenken, eigene Verhaltensweisen, Einstellungen, Normen, soziale Unterstützung und psychologische Sicherheit am Arbeitsplatz erfasst. Im zweiten Teil wurden vier Vignetten präsentiert, die von den Befragten zu beurteilen waren. In diesen kurzen Fallgeschichten war jeweils eine hypothetische Situation geschildert, in der ein Kollege einen Fehler macht oder eine Sicherheitsregel verletzt. In die vier «Rahmenhandlungen» waren jeweils Details eingebettet, die in einem voll-faktoriellen Versuchsplan systematisch manipuliert worden waren. Die Rahmenhandlungen waren aus den Interviews der ersten Studienphase inspiriert und beinhalteten: einen übersehenen Verordnungsfehler; eine ausgelassene Hände-Desinfektion bei einer Wunduntersuchung; eine ausgelassene Doppelkontrolle von Medikamenten; die Durchführung einer Lumbalpunktion bei einem Patienten unter Chemotherapie ohne aktuelle Thrombozytenzahl und Gerinnungsstatus. Sieben Details wurden integriert, deren Ausprägungen variierten. Beispielsweise wurde die Handlung in einem Fall von einem leitenden Arzt, in einer anderen Variante von einem Assistenzarzt durchgeführt. Jeder Befragte erhielt randomisiert vier Vignetten. Die Befragten beurteilten jede Situation

Tabelle 1: In den Interviews häufig genannte Sicherheitsbedenken.

\begin{tabular}{ll}
\hline Häufige Sicherheitsbedenken & \\
\hline Gesamter Medikationsprozess & - Falsche Dosierung/Gesamtdosis verordnet \\
\hline & - Keine/falsche Prämedikation \\
\hline & - Falsche Durchlaufgeschwindigkeit Infusion \\
\hline & - Verordnungen falsch ausgefällt \\
\hline Hygiene/Isolation & - Fehlendes Verordnungsblatt für intrathekale \\
\hline & Therapie \\
\hline Behandlungsentscheide & - Keine Handschuhe/Händedesinfektion \\
\hline Invasive Prozeduren & - Prozeduren, die in Isolation nicht erlaubt sind \\
\hline & - Verwendung unsteriler Materialien \\
\hline & - Entscheid für OP \\
\hline & - Keine) Verlegung auf IPS \\
\hline & - Lumbalpunktion ohne Gerinnungsparameter \\
\hline
\end{tabular}

anhand eines Fragensets, zum Beispiel wie hoch sie das Schadenspotential für den Patienten einschätzen, und wie sie sich persönlich als Bystander in der Situation verhalten würden. An der schriftlichen Befragung nahmen 1013 Ärzte und Pflegefachpersonen teil.

\section{Resultate}

Unsere Untersuchung zeigt, dass Sicherheitsbedenken im Alltag häufig vorkommen. Die Hälfte der antwortenden Ärztinnen und Pflegefachpersonen gab an, mindestens manchmal einen Fehler zu bemerken, der gefährlich für Patienten sein könnte (z.B. eine ungenaue Verordnung). 37\% gaben an, dass sie mindestens manchmal mitbekommen, dass Kollegen sich bewusst oder unbewusst nicht an Regeln halten, die für die Patientensicherheit wichtig sein können (z.B. Doppelkontrollen oder Hygienerichtlinien). Dies zeigt eine hohe Aufmerksamkeit gegenüber sicherheitsrelevanten Ereignissen. In den Interviews wurden vielfältige Situationen benannt, die im Alltag Sicherheitsbedenken bei den Mitarbeitenden auslösen (Tab. 1) [2].

Das Zurückhalten von konkreten Sicherheitsbedenken und Verbesserungsvorschlägen ist kein seltenes Verhalten: Viele der Befragten gaben an, mindestens einmal

- ihre Gedanken oder Ideen zur Verbesserung der Patientensicherheit in ihrer Abteilung für sich behalten zu haben (74\%);

- entschieden zu haben, ihre Bedenken zur Patientensicherheit nicht zu äussern (72\%);

- Fragen zur Patientensicherheit in ihrer Abteilung lieber nicht gestellt zu haben (54\%);

- mögliche Sicherheitsprobleme in ihrer Abteilung festgestellt und anderen Personen nichts darüber gesagt zu haben (49\%);

- geschwiegen zu haben, obwohl ihre Hinweise möglicherweise eine Gefahr für Patienten reduziert hätten (38\%).

Das Ansprechen von Sicherheitsproblemen fällt bei manchen Themen leichter, bei anderen schwerer. Fast alle berichteten Episoden des «Schweigens» standen im Zusammenhang mit Hygienemassnahmen, Handlungen bei Patienten in Isolation und invasiven Prozeduren. Im Gegensatz dazu existiert eine gut etablierte Kultur, Zweifel und Hinweise hinsichtlich der Medikationssicherheit zu kommunizieren. Die meisten Situationen, in denen die Fachpersonen ihre Bedenken zurückhielten, wiesen gemeinsame, typische Merkmale auf: Es waren mehrere Personen anwesend (z.B. Visite) und es waren Hierarchie- oder Berufsgruppenunterschiede zwischen den Beteiligten vorhanden. Es 
wurden wenige Situation berichtet, in denen alle Beteiligten Pflegefachpersonen waren oder gleichrangige Ärztinnen und Ärzte.

Die Ergebnisse aus der schriftlichen Befragung bestätigten, dass die eigene hierarchische Position assoziiert ist mit der Häufigkeit des Zurückhaltens von Sicherheitsbedenken: Je höher die eigene hierarchische Position, umso eher werden Nachfragen gestellt und die Einhaltung von sicherheitsrelevanten Regeln angemahnt. Eine Regressionsanalyse zeigte, dass eine starke psychologische Sicherheit am Arbeitsplatz (sich durch Kollegen und Vorgesetzte unterstützt und fair behandelt fühlen) und ein hohes Mass an Patientensicherheits-Anwaltschaft (sich selber als Fürsprecher für die Patientensicherheit zu sehen) mit einer geringeren Frequenz des Schweigens verbunden sind [3]. Die Fachleute finden mehrheitlich, dass ein hohes Mass an interpersonellen, kommunikativen und CopingFähigkeiten erforderlich sind, um Sicherheitsbedenken und Zweifel auszusprechen: Es ist nichts, was den meisten Personen leicht fällt. In unserer Stichprobe sahen wir auch deutliche Unterschiede in der wahrgenommenen psychologischen Sicherheit zwischen den Abteilungen. Selbst bei Adjustierung der Mitarbeitermerkmale war dieser wichtige Faktor am höchsten bei den Mitarbeitenden pädiatrischer Onkologien. Offensichtlich gelingt es dort eher, eine Kultur und Kommunikationsstile zu etablieren, die das Ansprechen von heiklen Verhaltensweisen leichter macht. In den Interviews berichteten die Fachpersonen, verschiedene Kommunikationsarten einzusetzen, um ihren Bedenken Ausdruck zu verleihen, von denen viele nonverbaler Art sind. Jede zweite Person berichtete, Gesten und Mimik zu nutzen, um Kollegen Sicherheitshinweise zu signalisieren. Vor allem bei Verletzungen von Hygieneregeln ist dabei die Hauptmotivation, den Kollegen weniger blosszustellen, gerade wenn Patienten anwesend sind (s. Box). Besonders Mitarbeitende der pädiatrischen Onkologie versuchten oft, ihre Zweifel oder Bedenken diskret zu kommunizieren (z.B. Räuspern), um Eltern der behandelten Kinder nicht zu verunsichern. Fast alle Interview-Partner berichteten, dass es schwierig sei, die richtigen Worte zu finden, um Kollegen auf Sicherheitsprobleme anzusprechen. Dies führt in akuten Situationen oft dazu, dass nicht schnell genug reagiert werden kann, da es ganz einfach zu schnell geht, um sich diploma-

Zitat aus einem Interview:

«Dass man selber die Maske anzieht und schaut, ob er auch reagiert, oder sich selber eine anziehen und ihm die Sachen hinhalten. Vorschriften oder Guidelines hätten wir, die wären ja da.» (Assistenzärztin, onkologische Pädiatrie, Schweiz 2013) tische Formulierungen zu überlegen. Die Mitarbeitenden wählen die Formulierungen jeweils dem beteiligten Gegenüber entsprechend, versuchen diplomatische Gesprächsstile, und manchmal durchdachte Kommunikationstaktiken, insbesondere wenn sie Vorgesetzten ihre Sicherheitsbedenken mitteilen wollen. Assistenzärzte beispielsweise verpacken ihre Zweifel eher im Stil von Fragen und «stellen sich dumm», um den Vorgesetzten "mit der Nase drauf zu stupsen", auch wenn sie überzeugt sind, dass eine Gefährdung für Patienten besteht.

Der Entscheidung, Sicherheitsbedenken auszusprechen oder zurückzuhalten, geht oft eine komplexe Abwägung voraus [4]. Dabei steht der starken Motivation, Patienten vor potentiellem Schaden zu schützen, die Sorge vor schwierigen Situationen und Störungen guter kollegialer Beziehungen gegenüber. Besonders schwierig ist dieser Abwägungsprozess, wenn die Beteiligten sich nicht gut kennen und die Reaktion des Gegenübers dadurch schwer prognostizierbar ist. Explizit genannt wurden in den Interviews beispielsweise Situationen mit Konsiliarärzten oder auch Rotationsassistenten.

Bei den Vignetten im schriftlichen Fragebogen gaben die Teilnehmer mehrheitlich an, sie würden ihre Kollegen auf die riskante Situation bzw. die Regelverletzung hinweisen. Allerdings variierte diese Bereitschaft stark zwischen den Fallgeschichten, also je nach Rahmenhandlung, und innerhalb dieser je nach Ausprägung der geschilderten Details (z.B. Berufsgruppe der Beteiligten; wiederholtes Vorkommen des Vorfalls usw.) [5]. Je nachdem, wie diese Details ausgestaltet waren, würden

- 74-96\% gegenüber einem ärztlichen Vorgesetzten aussprechen, dass er einen Verordnungsfehler übersehen hat;

- 45-81\% einen Kollegen (Ärztin, Arzt oder Pflegefachperson) darauf hinweisen, dass er die Händedesinfektion vor einer Wundkontrolle vergessen hat;

- 82-94\% eine Pflegefachperson auf die übergangene Doppelkontrolle von Medikamenten ansprechen;

- 59-92\% das Verhalten eines ärztlichen Kollegen kritisch hinterfragen, der bei einer Lumbalpunktion die Sicherheitsvorgaben nicht einhält.

Durchschnittlich variierte die Bereitschaft, Sicherheitsbedenken vorzubringen, innerhalb einer Rahmenhandlung um 25\%, je nachdem, wie die Situation beschrieben war. Klar wurde auch, dass es vielen Fachpersonen unangenehm ist, Kollegen die eigenen Bedenken mitzuteilen. Über alle Vignetten hinweg gaben 31\% der Befragten an, dass es ihnen unangenehm wäre, den Kollegen auf die potentielle Gefahr hinzuweisen. 
Bei der ausgelassenen Händedesinfektion war dieser Anteil mit $42 \%$ am höchsten.

Der wichtigste Prädiktor für die Äusserung der eigenen Bedenken ist die Einschätzung des Schadenspotentials der Situation für den hypothetischen Patienten: Je gefährlicher eine Situation eingeschätzt wird, umso eher sind die Befragten bereit, sich persönlich zu exponieren und das Wort zu erheben. In den Beurteilungen der Fallgeschichten und dem antizipierten eigenen Verhalten zeigten sich deutliche Unterschiede zwischen Personen mit und ohne Leitungsfunktionen, einem Indikator für die hierarchische Stellung in der Abteilung: Befragte ohne Leitungsfunktionen gaben deutlich seltener an, ihre Bedenken in der geschilderten Situation äussern zu wollen, und fanden dies auch deutlich häufiger unangenehm. Interessant ist, dass das Schadenspotential der Situationen für den hypothetischen Patienten von Personen mit und ohne Leitungsfunktion und unabhängig von der Berufsgruppe etwa gleich eingeschätzt wurde. Wenn also rangtiefere Personen ihre Bedenken nicht äussern, dann liegt das nicht daran, dass sie nicht die Gefährdung erkennen würden (was beispielsweise über die kürzere klinische Erfahrung erklärbar wäre). Das Risiko wird von den verschiedenen Gruppen ähnlich eingeschätzt, aber die persönliche Bereitschaft, selber aktiv einzuschreiten, ist bei Personen ohne Führungsfunktion geringer, da sie eher interkollegiale Konflikte und gestörte Beziehungen fürchten.

Sowohl aus den Interviews als auch aus der Beantwortung der Vignetten ging hervor, dass eine Situation für die Fachleute am schwierigsten zu lösen ist: Zeuge einer riskanten Handlung zu sein, bei der der Patient anwesend und aufmerksam ist. Dies produziert einen erheblichen Entscheidungskonflikt, einerseits den Patienten vor möglichen Schädigungen schützen, und ihn andererseits nicht verunsichern und die Beziehung zum Behandlungsteam nicht gefährden zu wollen. Ein Dilemma, in dem die Abwägung schwierig ist und in dem - gerade wenn es schnell gehen muss häufig für das Schweigen entschieden wird.

\section{Schlussfolgerung}

Diese Studie zeigt, dass viele Mitarbeitende Sicherheitsbedenken im klinischen Alltag kennen. Ihnen fallen wichtige, sicherheitsrelevante Situationen und Verhaltensweisen der Kollegen auf. Damit sind die Mitarbeitenden ein wichtiges Sicherheitspotential. Die meisten Ärztinnen, Ärzte und Pflegefachpersonen haben in der
Vergangenheit Erfahrungen mit beiden Verhaltensweisen gemacht: ihre Bedenken, Fragen und Zweifel auszusprechen, aber auch sie zurückzuhalten. Das Schweigen ist erklärbar durch individuelle Faktoren (Alter, Funktion, Persönlichkeit), organisationelle Faktoren (psychologische Sicherheit, Hierarchie), und vor allem auch situative Kontextfaktoren (Thema, Beteiligte, Patienten, Risikoeinschätzung). Individuelle $\mathrm{Pa}$ tienten vor vermeidbarem Schaden zu schützen, ist die dominierende und starke Motivation für Mitarbeitende, auch persönlich unangenehme und als riskant wahrgenommene Situationen in Kauf zu nehmen. Problematisch ist, dass das Ansprechen von Sicherheitsproblemen über die akute Situation hinaus noch sehr selten stattfindet. Der Wert von "speaking up» wird noch nicht als Lern- oder Verbesserungschance über den Einzelfall hinaus gesehen. Mitarbeitende im Spital, gerade jene in weniger ranghohen Positionen, benötigen klare Signale der Führung, dass «speaking up» gewünscht und erwartet ist. Da sich sicherheitsrelevante Situationen oft sehr schnell entwickeln, und meist keine Zeit ist, sich erst dann die "passenden Worte» zu überlegen, kann es hilfreich sein, innerhalb einer Abteilung oder Praxis Formulierungen, Gesten und Signalwörter zu vereinbaren, die akzeptiert sind und von niemandem als verletzend oder angreifend verstanden werden. Nur wenn Fehler, Sicherheitsbedenken und riskante Verhaltensweisen in Teams offen und konsequent angesprochen werden, können Verbesserungen für die Patientensicherheit erzielt werden.

\section{Danksagung}

Wir danken allen Pflegefachpersonen und Ärztinnen und Ärzten, die an der Befragung oder den Interviews teilgenommen haben. Den Chefärztinnen und Chefärzten und den Pflegedienstleitungen der beteiligten onkologischen Abteilungen gilt unser besonderer Dank für ihre Offenheit, diese Studie zu unterstützen. Die Studie wurde massgeblich durch die Forschungsförderung der Krebsforschung Schweiz finanziert [KFS-2974-08-2012].

Literatur

1 Okuyama A, Wagner C, Bijnen B. Speaking up for patient safety by hospital-based health care professionals: a literature review. BMC Health Serv Res. 2014;14(1):61.

2 Schwappach DLB, Gehring K. «Saying it without words»: a qualitative study of oncology staff's experiences with speaking up about safety concerns. BMJ Open. 2014;4(5):e004740.

3 Schwappach DLB, Gehring K. Frequency of and predictors for withholding patient safety concerns among oncology staff: a survey study. Eur J Cancer Care. 2015;24(3):395-403.

4 Schwappach D, Gehring K. Trade-offs between voice and silence: a qualitative exploration of oncology staff's decisions to speak up about safety concerns. BMC Health Serv Res. 2014;14(1):303.

5 Schwappach DLB, Gehring K. Silence That Can Be Dangerous: A Vignette Study to Assess Healthcare Professionals' Likelihood of Speaking up about Safety Concerns. PLoS ONE. 2014;9(8):e104720. 